\title{
Type-2 Combined T-S Adaptive Fuzzy Control
}

\author{
Yunli Hao, ${ }^{1}$ Shuang Li $\mathbb{D}^{1},{ }^{1}$ Qing Xia, ${ }^{1}$ and Maohua Wang ${ }^{2}$ \\ ${ }^{1}$ School of Management, China University of Mining and Technology, Jiangsu 221116, China \\ ${ }^{2}$ College of Information Engineering, Fuyang Normal University, Anhui 236041, China
}

Correspondence should be addressed to Shuang Li; liprofessorcumt@163.com

Received 5 November 2019; Revised 19 March 2020; Accepted 23 April 2020; Published 15 May 2020

Academic Editor: Xinggang Yan

Copyright $\odot 2020$ Yunli Hao et al. This is an open access article distributed under the Creative Commons Attribution License, which permits unrestricted use, distribution, and reproduction in any medium, provided the original work is properly cited.

For a class of nonlinear systems with a nonlinear relationship between input and output, a fuzzy control method combining interval type- 2 and T-S fuzzy controller is proposed based on type- 2 fuzzy system theory. In order to ensure its stability, antiinterference ability, and minimum approximation error, this design combines direct, indirect, supervised, and compensation control types to construct the controller. In this way, the structure of the controller not only has the characteristics of the type- 2 fuzzy set, which can reduce the uncertainty of rules, but also has a T-S fuzzy model with linear combination of input variables, which can improve the modeling accuracy and reduce the number of rules of the system. By using the Lyapunov synthesis method, the global stability and the convergence of the closed-loop system under the condition that all variables are uniformly bounded are analyzed, and the adaptive laws of the system parameters are given as well. Finally, the effectiveness and superiority of the proposed method are verified by simulation.

\section{Introduction}

In recent years, fuzzy control has developed rapidly. However, to overcome the complexity and uncertainty of the system, the design of the controller needs higher requirements. The fuzzy system [1] has a powerful ability to cope with nonlinear and ambiguity problems since fuzzy logic itself provides the construction of linguistic information by experts. At present, it has become a systematic reasoning method widely used in the study of control strategies.

In industrial control, the control object is often time varying and nonlinear [2]. Due to the pure lag, unknown parameters, and drift, the mathematical model is difficult to be established. Fuzzy theory can deal with many fuzzy problems in industrial production, which provide a means to improve the control. In [3], Prof. Mamdani first applied the fuzzy control theory into the control of the steam engine and boiler and achieved better control effect than the conventional controller, which created a precedent for the successful application of fuzzy control theory in engineering. Subsequently, many scholars applied the fuzzy theory to automatic control systems such as temperature control, digital image stabilization, washing machine, automobile speed, and subway $[4,5]$. Since Takagi and Sugeno proposed a new fuzzy model, i.e., T-S model [5] in 1985, this model has become the focus of many experts and scholars because it can be designed easily and has better approximation performance than the Mamdani-type model [6]. In 1992, Siemens added fuzzy control software to the original process control system of automatic instrument and adopted the method of combining software and hardware. The first fuzzy logic control system was developed by Mamdani for a small steam engine [7]. Since then, the fuzzy logic control system has been widely used in industrial systems and consumer goods, which has attracted the attention of many researchers.

Because the nonlinear and random disturbance of the controlled object is difficult to describe and control, in actual industrial production [8-10], for some complex nonlinear systems, the general fuzzy controller cannot achieve satisfactory control effect. In $[8,9]$, direct and indirect adaptive fuzzy control methods are proposed, but the proposed supervisory control term is discontinuous, which may lead to sawtooth oscillation near the boundary. And the convergence of the tracking error depends on the strict condition that the minimum approximation error satisfies the square 
integrable. The combined fuzzy control method has been widely used to solve the control problems of various complex nonlinear systems [10]. The combined adaptive fuzzy controller combines the direct and indirect controllers by adjusting the factors. It uses the prediction error and track error to design the adaptive law of the system, which improves the convergence speed of the system. The traditional Mamdani-type combined adaptive control [11] only designs the controller according to the current state of the system and does not further predict its development trend. The T-S fuzzy control makes up for this shortcoming. In particular, the machine theory which combines the fuzzy control with other intelligent methods is of great significance in the research of complex nonlinear systems. For example, in 2002, Mehrdad Hojati introduced the identification model into the traditional T-S model [11], from which the model error of the system was obtained, and the adaptive laws were given by combining model error with tracking error. In this study, this method was applied to the design of the dragonfly model [12], and good results were obtained.

Type-1 fuzzy system has powerful nonlinear system identification and control capabilities. It is simple to implement and solves many problems in the control field. However, the membership function limits the ability of fuzzy sets to describe uncertainty. Therefore, type- 1 fuzzy system is established. Zadeh extended the type-1 fuzzy set theory in 1975 to form a new type-2 fuzzy set theory $[13,14]$. The membership of this set is ambiguous and can describe the limited shortcomings of the fuzzy problems. Since then, many scholars further studied the type- 2 fuzzy set. Firstly, other studies proposed the concept of the interval type- 2 fuzzy set and used it as the input or output of the system [15-17]. This improvement reduces the computational complexity of type-2 fuzzy sets, enhances the real-time application ability of type- 2 fuzzy systems, and promotes the application of type- 2 fuzzy set theory. By selecting different defuzzifiers to discuss the design of interval type-2 fuzzy systems, some researchers improved the theory of interval type- 2 fuzzy systems, and the parameters of interval type- 2 fuzzy systems are trained [18-20]. Some others pointed out the research status and development prospects of type-2 fuzzy systems in $[11,21]$.

Because the T-S fuzzy model has great advantages in guaranteeing universal approximation and system stability, in recent years, studies on the type- 2 T-S fuzzy model control achieved rich results [13-25]. In this paper, the type-2 direct adaptive fuzzy control of Mamdani and T-S type is used for addressing nonlinear systems, i.e., [26-28]. The proposed method ensures the stability and convergence of the closedloop system and achieves good results in solving the uncertainty and the influence of noise on the system effect. The design method of the interval type-2 T-S indirect adaptive fuzzy controller has been proposed in $[29,30]$. The rule front of the system is the interval type- 2 fuzzy set, and the latter is the exact number, which makes the control method of the structure. It not only has the characteristics of type- 2 fuzzy set processing but also can reduce the influence of rule uncertainty on the system. At the same time, it has the characteristics of linear combination of input variables in the T-S fuzzy model [31-33], which can improve the system modeling accuracy, reduce the number of rules, and so on. However, this control system requires that the approximation error satisfies the square integrable [34], and the supervisory control term is continuous. Its anti-interference ability is also strong [35].

In order to achieve better control performance (i.e., improve the modeling accuracy and reduce the number of rules of the system), the research of combined controller is of great significance. To the best of authors' knowledge, the type- 2 combined T-S fuzzy model is still not studied, which is an open issue and full of challenges for them. Therefore, based on the above discussion, this paper proposes a type- 2 combined T-S fuzzy control with continuous supervisory control capability which is proposed for a class of uncertain nonlinear systems with unknown functions. The combined design of the controller is divided into direct, indirect, supervisory, and adaptive compensation control items. Direct and indirect control items are used to obtain the desired tracking performance. Supervisory item can ensure that the system states are bounded so as to improve the dynamic performance of the system. The adaptive compensation term can compensate the influence of the approximation error and external interference. Due to the complexity of the control algorithm, type-2 fuzzy control is difficult to be applied online. The realization process of the proposed method is as follows: (1) a fuzzy system is designed for system (1) to approximate its unknown nonlinear function. (2) Using the KM iterative method, the fuzzy reasoning analysis (10)-(23) is carried out accurately. (3) Construction of a controller (25). (4) Design the adaptive parameters (42)-(45). This control method can update the adaptive laws online so as to realize the online control for system (1), and make the tracking error $e \longrightarrow 0$ as $t \longrightarrow \infty$.

The main contributions of this paper are as follows:

(1) Compared with papers [27-29], this paper adopts the combination of direct control, indirect control, supervisory control, and adaptive compensation. It has strong performance in tracking unmeasurable system state performance, ensures boundedness of the system states, improves the dynamic performance of the system, and compensates the approximation error and external disturbance.

(2) The adaptive laws can adjust online and improve the tracking accuracy of the controlled object, which makes the system to have many perfect characteristics in dealing with uncertainty. It shows more effective than [31, 32] in dealing with unknown disturbance and training noise. 
(3) It has strong anti-interference ability and can effectively alleviate the chattering phenomenon commonly seen in the sliding mode control system, such as the chattering problem in [34]. Aiming at the problem with robustness in [33-35], the robustness of the system against disturbances and uncertainties and the convergence rate can be effectively improved so as to make the system have active adaptability and better stability.

The simulation results show that the designed controller can achieve a good control effect by using less fuzzy rules, which can make the system state variables be with smaller fluctuation and faster convergence under the random disturbance, which shows that the new controller has stronger anti-interference ability and advantages.

\section{Niche Control}

Consider the following order nonlinear system:

$$
\left\{\begin{array}{l}
x^{(n)}=f\left(x, \dot{x}, \ldots, x^{(n-1)}\right)+g\left(x, \dot{x}, \ldots, x^{(n-1)}\right) u+d(t), \\
y=x
\end{array}\right.
$$

Here, $f$ and $g$ are unknown but bounded functions, $u, y \in R$ are the input and output of the system, respectively, $y$ and $d(t)$ are always bounded perturbations. It is assumed here that not all $x_{i}$ are measurable. In order to make (1) measurable, we ask $g(X) \neq 0$ acts, $X=\left(x_{1}, \ldots, x_{n}\right)^{T}=\left(x, \dot{x}, \ldots, x^{(n-1)}\right)^{T} \in R^{n}$. Some $X$ are in a controllable interval $U_{c} \subset R^{n}$. Because $g(X)$ is continuous, we suppose $0<g(X) \leq \infty$ under the circumstance $X \in U_{c}$. The control objective is to design a hybrid controller, adjust the relevant parameters, and get the adaptive law of the parameters and make the output $y$ of the system track a related bounded reference signal $y_{m}(t)$ under a tight set. All signals are bounded. Initially, the signal vector $\bar{y}_{m}(t)$, tracking error vector $e$, and estimated error vector $\bar{e}$ are defined as

$$
\begin{aligned}
\bar{y}_{m} & =\left(y_{m}, \dot{y}_{m}, \ldots, \dot{y}_{m}{ }^{(n-1)}\right)^{T} \in R^{n}, \\
e & =y_{m}-X=\left(e, \dot{e}, \ldots, e^{(n-1)}\right)^{T} \in R^{n}, \\
\bar{e} & =\bar{y}_{m}-\bar{X}=\left(\bar{e}, \dot{\bar{e}}, \ldots, \bar{e}^{(n-1)}\right)^{T} \in R^{n} .
\end{aligned}
$$

Here, $\bar{X}$ and $\bar{e}$ are $X$ and $e$ estimates. If $f(X)$ and $g(X)$ are known and the system is free $d(t)$ to external disturbances, at this point, we can choose the controller $u^{*}$ to eliminate the nonlinearity and design the controller. Define $k_{0}=\left(k_{01}, \ldots, k_{0 n}\right)^{T} \in R^{n}$, and let $s^{n}+k_{0 n} s^{n-1}+\cdots+k_{01}$. All the roots of them are on the left half-open plane of the complex plane, and one can get the following ideal controller:

$$
u^{*}=\frac{1}{g(X)}\left[-f(x)+y_{m}^{(n)}+k_{0}{ }^{T} e\right] .
$$

Substituting (3) into (1), we can get the maximum control target $\lim _{t \longrightarrow \infty} e(t)=0$, but $f(X)$ and $g(X)$ are unknown, ideal controller (3) cannot be implemented, and not all system states can be measured. So, we have to set 2 . description of the T-S fuzzy system.

Since $f(X)$ and $g(X)$ are unknown functions, the interval type-2 T-S fuzzy system is used to approximate them. The type- 2 combined T-S fuzzy rules are described as follows:

$$
\begin{aligned}
& R_{f}^{l}: \text { if } x_{1} \text { is } A_{1}^{l} \text { and } \ldots \text { and } x_{n} \text { is } A_{n}^{l}, \quad \text { so, } \\
& \widehat{f}(X)=a_{0}^{l}+a_{1}^{l} x_{1}+\cdots+a_{n}^{l} x_{n}, \\
& R_{g}^{l}: \text { if } x_{1} \text { is } G_{1}^{l} \text { and } \ldots \text { and } x_{n} \text { is } G_{n}^{l}, \quad \text { so, } \\
& \widehat{g}_{l}(X)=b_{0}^{l}+b_{1}^{l} x_{1}+\cdots+b_{n}^{l} x_{n}, \\
& R_{u}^{l}: \text { if } x_{1} \text { is } U_{1}^{l} \text { and } \ldots \text { and } x_{n} \text { is } U_{n}^{l}, \quad \text { so, } \\
& \widehat{u}_{l}(X)=c_{0}^{l}+c_{1}^{l} x_{1}+\cdots+c_{n}^{l} x_{n}, \quad(l=1, \ldots m) .
\end{aligned}
$$

Here, $A_{i}^{l}, G_{i}^{l}$, and $U_{i}^{l}$ are state vector $x_{i}$. The interval type2 fuzzy set of $s$ selects the Gaussian membership function and satisfies $x_{i}^{l}-x_{j}^{l} \gg \sigma$, here $x_{i}^{l}, x_{j}^{l}$, and $\sigma$ are the centers of membership, and the width $d$ is the constant coefficient of the state vector of the type-2 fuzzy system. $a_{k}^{l}, b_{k}^{l}$, and $c_{k}^{l}$ are the constant coefficients of the state vector of the type- 2 fuzzy system, and the predecessor is an interval type-2 type fuzzy set. The rule front of the fuzzy system $\widehat{f}(X)$ is an interval type-2 fuzzy set whose membership functions are

$$
\begin{aligned}
\rho_{A_{1}^{l} \cdot A_{2}^{l} \cdots A_{n}^{l}}(X) & =\rho_{A_{1}^{l}}\left(x_{1}\right) \cdot \rho_{A_{2}^{l}}\left(x_{2}\right) \cdots \rho_{A_{n}^{l}}\left(x_{n}\right), \\
& =\prod_{l=1}^{n} \rho_{A_{k}^{l}}\left(x_{k}\right) k=(1,2 \cdots, n) .
\end{aligned}
$$

Gaussian membership function:

$$
\rho_{A_{i}^{l}}\left(x_{l}\right)=\exp \left(-\left(\frac{x_{i}-\bar{x}_{i}^{l}}{\sigma_{i}^{l}}\right)^{2}\right), \quad i=(1,2 \cdots, n) \text {. }
$$

Output mixed type-2 type fuzzy set can be represented by $\widetilde{Z}_{f}^{i}, i=(1,2, \cdots, M)$, and using the center average deblurring, the down type is

$$
\widehat{F}\left(\widetilde{Z}_{f}^{1}, \cdots, \widetilde{Z}_{f}^{M}\right)=\int_{z_{f}^{1}} \cdots \int_{z_{f}^{M}} \tau_{i=1}^{M} \rho_{\widetilde{Z}^{i}}\left(\widetilde{z}^{i}\right) / \frac{\sum_{i=1}^{M} z_{f}^{i} \widehat{f}^{i}(X)}{\sum_{i=1}^{M} z_{f}^{i}} .
$$

Using the membership functions for $A_{k}^{i}$, then (7) becomes

$$
\widehat{F}\left(\widetilde{Z}_{f}^{1}, \cdots, \widetilde{Z}_{f}^{M}\right)=\int_{z_{f}^{1}} \cdots \int_{z_{f}^{M}} 1 / \frac{\sum_{i=1}^{M} z_{f}^{i} \widehat{f}^{i}(X)}{\sum_{i=1}^{M} z_{f}^{i}},
$$


where

$$
\begin{aligned}
\widehat{F} & =\left[\hat{f}_{l}, \hat{f}_{r}\right], z_{f}^{i} \in \widetilde{Z}_{f}^{i} \in\left[\underline{z}_{f}^{i}, \bar{z}_{f}^{i}\right], \\
\underline{z}_{f}^{i} & =\rho_{\underline{A}_{1}^{i}}\left(x_{1}\right) \cdot \rho_{\underline{A}_{2}^{i}}\left(x_{2}\right) \cdots \rho_{\underline{A}_{n}^{i}}\left(x_{n}\right), \\
\bar{z}_{f}^{i} & =\rho_{\bar{A}_{1}^{i}}\left(x_{1}\right) \cdot \rho_{\bar{A}_{2}^{i}}\left(x_{2}\right) \cdots \rho_{\bar{A}_{n}^{i}}\left(x_{n}\right),
\end{aligned}
$$

where $\tau$ and . are the small or product $t$-paradigms.

Using the KM iterative method [26], we can see that

$$
\begin{aligned}
\widehat{f}_{l} & =\frac{\sum_{i=1}^{L} \bar{z}_{f}^{i} \widehat{f}^{i}+\sum_{j=L+1}^{M} \underline{z}_{f}^{j} \widehat{f}^{j}}{\sum_{i=1}^{L} \bar{z}_{f}^{i}+\sum_{j=L+1}^{M} \underline{z}_{f}^{j}}, \\
& =\sum_{i=1}^{L} q_{f l}^{i} \widehat{f}^{i}+\sum_{j=L+1}^{M} q_{f l}^{j} \hat{f}^{j}, \\
& =\sum_{i=1}^{L} q_{f l}^{i} \theta_{f l}^{i} \vec{X}+\sum_{j=L+1}^{M} q_{f l}^{j} \theta_{f l}^{j} \vec{X}, \\
& =\left(\underline{Q}_{f l} \bar{Q}_{f l}\right)\left(\begin{array}{c}
\underline{\theta}_{f l} \\
\bar{\theta}_{f l}
\end{array}\right) \vec{X}, \\
& =\xi_{f l} \theta_{f l} \vec{X},
\end{aligned}
$$

where

$$
\begin{aligned}
& q_{f l}^{i}=\frac{\bar{z}_{f}^{i}}{\sum_{i=1}^{L} \bar{z}_{f}^{i}+\sum_{j=L+1}^{M} \underline{z}_{f}^{i}}, \\
& q_{f l}^{j}=\frac{\bar{z}_{f}^{j}}{\sum_{i=1}^{L} \bar{z}_{f}^{i}+\sum_{j=L+1}^{M} \underline{z}_{f}^{i}}, \\
& \underline{Q}_{f l}=\left(q_{f l}^{1}, q_{f l}^{2}, \cdots, q_{f l}^{L}\right) \text {, } \\
& \overline{\mathrm{Q}}_{f l}=\left(q_{f l}^{L+1}, q_{f l}^{L+2}, \cdots, q_{f l}^{M}\right) \text {, } \\
& \underline{\theta}_{f l}=\left(\begin{array}{lll}
\theta_{f l}^{1} & \cdots & \theta_{f l}^{L}
\end{array}\right)^{T}, \\
& \bar{\theta}_{f l}=\left(\begin{array}{lll}
\theta_{f l}^{L+1} & \cdots & \theta_{f l}^{M}
\end{array}\right)^{T}, \\
& \xi_{f l}=\left(\underline{Q}_{f l} \bar{Q}_{f l}\right), \\
& \theta_{f l}^{i}=\left(a_{l 0}^{i}, a_{l 1}^{i}, \cdots, a_{\mathrm{ln}}^{i}\right) \in R^{1 \times(n+1)}, \quad i=1,2, \cdots, L, \\
& \theta_{f l}^{j}=\left(a_{l 0}^{j}, a_{l 1}^{j}, \cdots, a_{\mathrm{ln}}^{j}\right) \in R^{1 \times(n+1)}, \quad j=L+1, \cdots, M, \\
& \vec{X}=\left(1, x_{1}, \cdots, x_{n}\right) \text {, } \\
& \widehat{f}_{r}=\frac{\sum_{i=1}^{R} \underline{z}_{f}^{i} \widehat{f}^{i}+\sum_{j=R+1}^{M} \bar{z}_{f}^{j} \widehat{f}^{j}}{\sum_{i=1}^{R} \underline{z}_{f}^{i}+\sum_{j=R+1}^{M} \bar{z}_{f}^{j}}, \\
& =\sum_{i=1}^{R} q_{f r}^{i} \hat{f}^{i}+\sum_{j=R+1}^{M} q_{f r}^{j} \hat{f}^{j}, \\
& =\sum_{i=1}^{R} q_{f r}^{i} \theta_{f r}^{i} \vec{X}+\sum_{j=R+1}^{M} q_{f r}^{j} \theta_{f r}^{j} \vec{X}, \\
& =\left(\begin{array}{ll}
\underline{Q}_{f l} & \bar{Q}_{f r}
\end{array}\right)\left(\begin{array}{c}
\underline{\theta}_{f r} \\
\bar{\theta}_{f r}
\end{array}\right) \vec{X}, \\
& =\xi_{f r} \theta_{f r} \vec{X}
\end{aligned}
$$

where

$$
\begin{aligned}
& q_{f r}^{i}=\frac{\underline{z}_{f}^{i}}{\sum_{i=1}^{R} \underline{z}_{f}^{i}+\sum_{j=R+1}^{M} \bar{z}_{f}^{j}}, \\
& q_{f r}^{j}=\frac{\bar{z}_{f}^{j}}{\sum_{i=1}^{R} \underline{z}_{f}^{i}+\sum_{j=R+1}^{M} \bar{z}_{f}^{j}},
\end{aligned}
$$$$
\underline{Q}_{f r}=\left(q_{f r}^{1}, q_{f r}^{2}, \cdots, q_{f r}^{R}\right),
$$$$
\bar{Q}_{f r}=\left(q_{f r}^{R+1}, q_{f r}^{R+2}, \cdots, q_{f r}^{M}\right)
$$$$
\underline{\theta}_{f r}=\left(\begin{array}{lll}
\theta_{f r}^{1} & \cdots & \theta_{f r}^{R}
\end{array}\right)^{T},
$$$$
\bar{\theta}_{f r}=\left(\begin{array}{lll}
\theta_{f r}^{R+1} & \cdots & \theta_{f r}^{M}
\end{array}\right)^{T}, \xi_{f r}=\left(\begin{array}{ll}
Q_{f r} & \bar{Q}_{f r}
\end{array}\right),
$$$$
\theta_{f r}^{i}=\left(a_{r 0}^{i}, a_{r 1}^{i}, \cdots, a_{r n}^{i}\right) \in R^{1 \times(n+1)}, \quad i=1,2, \cdots, R,
$$$$
\theta_{f r}^{j}=\left(a_{r 0}^{j}, a_{r 1}^{j}, \cdots, a_{r n}^{j}\right) \in R^{1 \times(n+1)}, \quad j=R+1, \cdots, M,
$$$$
\vec{X}=\left(1, x_{1}, \cdots, x_{n}\right)
$$$$
a_{k}^{i}=\left(\begin{array}{ll}
a_{l k}^{i} & a_{r k}^{i}
\end{array}\right)^{T}
$$$$
a_{k}^{j}=\left(\begin{array}{ll}
a_{l k}^{j} & a_{r k}^{j}
\end{array}\right)^{T},
$$$$
a_{k}=\left(\begin{array}{ll}
a_{k}^{i} & a_{k}^{j}
\end{array}\right)^{T}
$$$$
k=1,2, \cdots, n \text {. }
$$

Using average deblurring, there are (10) and (11) available for this type-2 fuzzy system output as

$$
\begin{aligned}
\hat{f}\left(X / \theta_{f l}, \theta_{f r}\right) & =\frac{\hat{f}_{l}+\hat{f}_{r}}{2}, \\
& =\frac{1}{2}\left(\xi_{f l} \theta_{f l}+\xi_{f r} \theta_{f r}\right) \vec{X}, \\
& =\frac{1}{2} \xi_{f} \theta_{f},
\end{aligned}
$$

where

$$
\begin{aligned}
& \xi_{f}=\left(\begin{array}{ll}
\xi_{f l} & \xi_{f r}
\end{array}\right) \vec{X} \\
& \theta_{f}=\left(\begin{array}{ll}
\theta_{f l} & \theta_{f r}
\end{array}\right)^{T} .
\end{aligned}
$$

The same reason: 
Mathematical Problems in Engineering

5

$$
\begin{aligned}
\widehat{g}_{l} & =\frac{\sum_{i=1}^{L^{1}} \underline{z}_{g}^{i} \widehat{g}^{i}+\sum_{j=L^{1}+1}^{M} \bar{z}_{g}^{j} \widehat{g}^{j}}{\sum_{i=1}^{L^{1}} \underline{z}_{g}^{i}+\sum_{j=L^{1}+1}^{M} \bar{z}_{g}^{j}}, \\
& =\sum_{i=1}^{L^{1}} q_{g l}^{i} \hat{g}^{i}+\sum_{j=L^{1}+1}^{M} q_{g l}^{j} \widehat{g}^{j}, \\
& =\sum_{i=1}^{L^{1}} q_{g l}^{i} \theta_{g l}^{i} \vec{X}+\sum_{j=L^{1}+1}^{M} q_{g l}^{j} \theta_{g l}^{j} \vec{X}, \\
& =\left(\underline{Q}_{g l} \bar{Q}_{g l}\right)\left(\begin{array}{c}
\underline{\theta}_{g l} \\
\bar{\theta}_{g l}
\end{array}\right) \vec{X}, \\
& =\xi_{g l} \theta_{g l} \vec{X},
\end{aligned}
$$

where

$$
\begin{aligned}
& q_{g l}^{i}=\frac{\underline{z}_{g}^{i}}{\sum_{i=1}^{L^{1}} \underline{z}_{g}^{i}+\sum_{j=L^{1}+1}^{M} \bar{z}_{g}^{i}}, \\
& q_{g l}^{j}=\frac{\underline{z}_{g}^{j}}{\sum_{i=1}^{L^{1}} \underline{z}_{g}^{i}+\sum_{j=L^{1}+1}^{M} \bar{z}_{g}^{i}}, \\
& \underline{Q}_{g l}=\left(q_{g l}^{1}, q_{g l}^{2}, \cdots, q_{g l}^{L^{1}}\right), \\
& \bar{Q}_{g l}=\left(q_{g l}^{L^{+}}, q_{g l}^{L^{1}+2}, \cdots, q_{g l}^{M}\right), \\
& \theta_{g l}^{i}=\left(b_{l 0}^{i}, b_{l 1}^{i}, \cdots, b_{\ln }^{i}\right) \in R^{1 \times(n+1)}, \quad i=1,2, \cdots, L^{1}, \\
& \theta_{g l}^{j}=\left(b_{l 0}^{j}, b_{l 1}^{j}, \cdots, b_{\ln }^{j}\right) \in R^{1 \times(n+1)}, \quad j=L^{1}+1, \cdots, M, \\
& \underline{\theta}_{g l}=\left(\begin{array}{lll}
\theta_{g l}^{1} & \cdots & \theta_{g l}^{L^{1}}
\end{array}\right)^{T}, \\
& \bar{\theta}_{g l}=\left(\begin{array}{lll}
\theta_{g l}^{L^{1}+1} & \cdots & \theta_{g l}^{M}
\end{array}\right)^{T}, \\
& \widehat{g}_{r}=\frac{\sum_{i=1}^{R^{1}} \underline{z}_{g}^{i} \hat{g}^{i}+\sum_{j=R^{1}+1}^{M} \bar{z}_{g}^{j} \widehat{g}^{j}}{\sum_{i=1}^{R^{1}} \underline{z}_{g}^{i}+\sum_{j=R^{1}+1}^{M} \bar{z}_{g}^{j}}, \\
& =\sum_{i=1}^{R^{1}} q_{g r}^{i} \hat{g}^{i}+\sum_{j=R^{1}+1}^{M} q_{g r}^{j} \widehat{g}^{j}, \\
& =\sum_{i=1}^{R^{1}} q_{g r}^{i} \theta_{g r}^{i} \vec{X}+\sum_{j=R^{1}+1}^{M} q_{g r}^{j} \theta_{g r}^{j} \vec{X} \\
& =\left(\begin{array}{ll}
\underline{Q}_{g r} & \bar{Q}_{g r}
\end{array}\right)\left(\begin{array}{c}
\underline{\theta}_{g r} \\
\bar{\theta}_{g r}
\end{array}\right) \vec{X}, \\
& =\xi_{g r} \theta_{g r} \vec{X}
\end{aligned}
$$

$$
\begin{aligned}
& q_{g r}^{i}=\frac{\underline{z}_{g}^{i}}{\sum_{i=1}^{R^{1}} \underline{z}_{g}^{i}+\sum_{j=R^{1}+1}^{M} \bar{z}_{g}^{j}}, \\
& q_{g r}^{j}=\frac{\bar{z}_{g}^{j}}{\sum_{i=1}^{R^{1}} \underline{z}_{g}^{i}+\sum_{j=R^{1}+1}^{M} \bar{z}_{g}^{j}}, \\
& \underline{Q}_{g r}=\left(q_{g r}^{1}, q_{g r}^{2}, \cdots, q_{g r}^{R^{1}}\right), \\
& \bar{Q}_{g r}=\left(q_{g r}^{R^{1}+1}, q_{g r}^{R^{1}+2}, \cdots, q_{g r}^{M}\right), \\
& \underline{\theta}_{g r}=\left(\begin{array}{lll}
\theta_{g r}^{1} & \cdots & \theta_{g r}^{R^{1}}
\end{array}\right)^{T}, \\
& \bar{\theta}_{g r}=\left(\begin{array}{lll}
\theta_{g r}^{R^{1}+1} & \cdots & \theta_{g r}^{M}
\end{array}\right)^{T}, \\
& \xi_{g r}=\left(\underline{Q}_{g r} \bar{Q}_{g r}\right), \\
& \theta_{g r}^{i}=\left(b_{r 0}^{i}, b_{r 1}^{i}, \cdots, b_{r n}^{i}\right) \in R^{1 \times(n+1)}, \quad i=1,2, \cdots, R^{1}, \\
& \theta_{g r}^{j}=\left(b_{r 0}^{j}, 0 b_{r 1}^{j}, \cdots, b_{r n}^{j}\right) \in R^{1 \times(n+1)}, \quad j=R^{1}+1, \cdots, M, \\
& b_{k}^{i}=\left(\begin{array}{ll}
b_{l k}^{i} & b_{r k}^{i}
\end{array}\right)^{T}, \\
& b_{k}^{j}=\left(\begin{array}{ll}
b_{l k}^{j} & b_{r k}^{j}
\end{array}\right)^{T}, \\
& b_{k}=\left(\begin{array}{ll}
b_{k}^{i} & b_{k}^{j}
\end{array}\right)^{T} \cdot k=1,2, \cdots, n .
\end{aligned}
$$

Using the average deblurring, there are (15) and (16) available for the type-2 fuzzy system output:

$$
\begin{aligned}
\hat{g}\left(X / \theta_{g l}, \theta_{g r}\right) & =\frac{\widehat{g}_{l}+\widehat{g}_{r}}{2}, \\
& =\frac{1}{2}\left(\xi_{g l} \theta_{g l}+\xi_{g r} \theta_{g r}\right) \vec{X}, \\
& =\frac{1}{2} \xi_{g} \theta_{g},
\end{aligned}
$$

where

$$
\begin{aligned}
& \xi_{g}=\left(\begin{array}{ll}
\xi_{g l} & \xi_{g r}
\end{array}\right) \vec{X}, \\
& \theta_{g}=\left(\begin{array}{ll}
\theta_{g l} & \theta_{g r}
\end{array}\right)^{T} \text {. }
\end{aligned}
$$

The same reason:

where 
6

Mathematical Problems in Engineering

$$
\begin{aligned}
\widehat{u}_{l} & =\frac{\sum_{i=1}^{L^{2}} \underline{z}_{u}^{i} \widehat{u}^{i}+\sum_{j=L^{2}+1}^{M} \bar{z}_{u}^{j} \widehat{u}^{j}}{\sum_{i=1}^{L^{2}} \underline{z}_{u}^{i}+\sum_{j=L^{2}+1}^{M} \bar{z}_{u}^{j}} \\
& =\sum_{i=1}^{L^{2}} q_{u l}^{i} \theta_{u l}^{i} \vec{X}+\sum_{j=L^{2}+1}^{M} q_{u l}^{j} \theta_{u l}^{j} \vec{X} \\
& =\left(\underline{Q}_{u l} \bar{Q}_{u l}\right)\left(\begin{array}{l}
\underline{\theta}_{u l} \\
\bar{\theta}_{u l}
\end{array}\right) \vec{X}, \\
& =\xi_{u l} \theta_{u l} \vec{X}
\end{aligned}
$$

where

$$
\begin{aligned}
& q_{u l}^{i}=\frac{\underline{z}_{u}^{i}}{\sum_{i=1}^{L^{2}} \underline{z}_{u}^{i}+\sum_{j=L^{2}+1}^{M} \bar{z}_{u}^{i}}, \\
& q_{u l}^{j}=\frac{\underline{z}_{u}^{j}}{\sum_{i=1}^{L^{2}} \underline{z}_{u}^{i}+\sum_{j=L^{2}+1}^{M} \bar{z}_{u}^{i}}, \\
& \underline{Q}_{u l}=\left(q_{u l}^{1}, q_{u l}^{2}, \cdots, q_{u l}^{L^{2}}\right), \\
& \bar{Q}_{u l}=\left(q_{u l}^{L^{2}+1}, q_{u l}^{L^{2}+2}, \cdots, q_{u l}^{M}\right) \\
& \underline{\theta}_{u l}=\left(\begin{array}{lll}
\theta_{u l}^{1} & \cdots & \theta_{u l}^{L^{2}}
\end{array}\right)^{T}, \\
& \bar{\theta}_{u l}=\left(\begin{array}{lll}
\theta_{u l}^{L^{2}+1} & \cdots & \theta_{u l}^{M}
\end{array}\right)^{T}, \\
& \theta_{u l}^{i}=\left(c_{l 0}^{i}, c_{l 1}^{i}, \cdots, c_{l n}^{i}\right) \in R^{1 \times(n+1)}, \quad i=1,2, \cdots, L^{2}, \\
& \theta_{u l}^{j}=\left(c_{l 0}^{j}, c_{l 1}^{j}, \cdots, c_{\ln }^{j}\right) \in R^{1 \times(n+1)}, \quad j=L^{2}+1, \cdots, M, \\
& \widehat{u}_{r}=\frac{\sum_{i=1}^{R^{2}} \underline{z}_{u}^{i} \widehat{u}^{i}+\sum_{j=R^{2}+1}^{M} \bar{z}_{u}^{j} \widehat{u}^{j}}{\sum_{i=1}^{R^{2}} \underline{z}_{u}^{i}+\sum_{j=R^{2}+1}^{M} \bar{z}_{u}^{j}}, \\
& =\sum_{i=1}^{R^{2}} q_{u r}^{i} \theta_{u r}^{i} \vec{X}+\sum_{j=R^{2}+1}^{M} q_{u r}^{j} \theta_{u r}^{j} \vec{X} \\
& =\left(\begin{array}{ll}
\underline{Q}_{u r} & \bar{Q}_{u r}
\end{array}\right)\left(\begin{array}{l}
\underline{\theta}_{u r} \\
\bar{\theta}_{u r}
\end{array}\right) \vec{X}, \\
& =\xi_{u r} \theta_{u r} \vec{X}
\end{aligned}
$$

$$
\begin{aligned}
& q_{u r}^{i}=\frac{\underline{z}_{u}^{i}}{\sum_{i=1}^{R^{2}} \underline{z}_{u}^{i}+\sum_{j=R^{2}+1}^{M} \bar{z}_{u}^{j}}, \\
& q_{u r}^{j}=\frac{\bar{z}_{u}^{j}}{\sum_{i=1}^{R^{2}} \underline{z}_{u}^{i}+\sum_{j=R^{2}+1}^{M} \bar{z}_{u}^{j}}, \\
& \underline{Q}_{u r}=\left(q_{u r}^{1}, q_{u r}^{2}, \cdots, q_{u r}^{R^{2}}\right) \\
& \bar{Q}_{u r}=\left(q_{u r}^{R^{2}+1}, q_{u r}^{R^{2}+2}, \cdots, q_{u r}^{M}\right) \\
& \underline{\theta}_{u r}=\left(\begin{array}{lll}
\theta_{u r}^{1} & \cdots & \theta_{u r}^{R^{2}}
\end{array}\right)^{T}, \\
& \bar{\theta}_{u r}=\left(\begin{array}{lll}
\theta_{u r}^{R^{2}+1} & \cdots & \theta_{u r}^{M}
\end{array}\right)^{T}, \\
& \xi_{u r}=\left(\underline{Q}_{u r} \bar{Q}_{u r}\right) \\
& \theta_{u r}^{i}=\left(c_{r 0}^{i}, c_{r 1}^{i}, \cdots, c_{r n}^{i}\right) \in R^{1 \times(n+1)}, \quad i=1,2, \cdots, R^{2}, \\
& \theta_{u r}^{j}=\left(c_{r 0}^{j}, c_{r 1}^{j}, \cdots, c_{r n}^{j}\right) \in R^{1 \times(n+1)}, \quad j=R^{2}+1, \cdots, M, \\
& c_{k}^{i}=\left(\begin{array}{ll}
c_{l k}^{i} & c_{r k}^{i}
\end{array}\right)^{T} \\
& c_{k}^{j}=\left(\begin{array}{ll}
c_{l k}^{j} & c_{r k}^{j}
\end{array}\right)^{T} \\
& c_{k}=\left(\begin{array}{cc}
c_{k}^{i} & c_{k}^{j}
\end{array}\right)^{T} \\
& k=1,2, \cdots, n .
\end{aligned}
$$

Using the average deblurring, there are (20) and (21) available for the type-2 fuzzy system output:

$$
\begin{aligned}
\widehat{u}\left(X / \theta_{u l}, \theta_{u r}\right) & =\frac{\widehat{u}_{l}+\widehat{u}_{r}}{2}, \\
& =\frac{1}{2}\left(\xi_{u l} \theta_{u l}+\xi_{u r} \theta_{u r}\right) \vec{X}, \\
& =\frac{1}{2} \xi_{u} \theta_{u},
\end{aligned}
$$

where

$$
\begin{aligned}
& \xi_{u}=\left(\begin{array}{ll}
\xi_{u l} & \xi_{u r}
\end{array}\right) \vec{X} \\
& \theta_{u}=\left(\begin{array}{ll}
\theta_{u l} & \theta_{u r}
\end{array}\right)^{T} .
\end{aligned}
$$

where 


\section{Design a Combined Controller with Supervisory and Compensation Control}

Construct a combined adaptive controller:

$$
u=\alpha u_{1}(\bar{X})+(1-\alpha) u_{D}(\overline{X \mid \theta})+u_{s}+u_{c} .
$$

Here, $u_{I}$ is an indirect controller, $u_{D}$ is the direct T-S model output controller, $u_{s}$ is the supervisory controller to constrain the state in the tight set, $\alpha \in[0,1]$ is the weighting factor, and $u_{c}$ is the adaptive control compensation controller. Replace the function $f(X), g(X)$, and the $e$ error vector with $\hat{f}\left(X_{-}\right), \hat{g}\left(X_{-}\right)$, and $e^{-}$, and the equivalent controller can be written as

$$
u^{*}=\frac{1}{g(x)}\left[-f(x)+y_{m}^{(n)}+k_{0}^{T} \bar{e}\right] .
$$

Indirect control law can be written as

$$
u_{I}=\frac{1}{g(\bar{X})}\left[-\widehat{f}(\bar{X})+y_{m}^{(n)}+k_{0}^{T} \bar{e}\right]
$$

and bringing (24) and (25) into (1), there is an error equation:

$$
\begin{aligned}
\dot{e}= & A e-B k_{0}^{T} \bar{e}+B\left\{\alpha\left[\hat{f}(\bar{X})-f(X)+(\hat{g}(\bar{X}) t-n g q(X)) u_{I}\right]\right\} \\
& +(1-\alpha) g(X)\left(u^{*}-u_{D}\right)+B g(X)\left(u_{s}+u_{c}\right)-B d .
\end{aligned}
$$

Let $k_{c}=\left(k_{c n}, \ldots K_{c 1}\right)$ and $\widetilde{e}=e-\bar{e}$; get

$$
\begin{aligned}
\dot{\tilde{e}}= & A \widetilde{e}-B \alpha \hat{f}(\bar{X})-f(X)+\hat{g}(\bar{X})-g(X) u_{I} \\
& +(1-\alpha) g(X)\left(u^{*}-u_{D}\right)-B g(X)\left(u_{s}+u_{c}\right)-B d,
\end{aligned}
$$

where

$$
\begin{aligned}
\Lambda=A-k_{c} C^{T} & =\left[\begin{array}{ccccccc}
-k_{c n} & 1 & 0 & 0 & \cdots & 0 & 0 \\
-k_{c(n-1)} & 0 & 1 & 0 & \cdots & 0 & 0 \\
\vdots & \vdots & \vdots & \vdots & \ddots & \vdots & \vdots \\
-k_{c 2} & 0 & 0 & 0 & \cdots & 0 & 1 \\
-k_{c 1} & 0 & 0 & 0 & \cdots & 0 & 0
\end{array}\right], \\
A & =\left[\begin{array}{ccccccc}
0 & 1 & 0 & 0 & \cdots & 0 & 0 \\
0 & 0 & 1 & 0 & \cdots & 0 & 0 \\
\vdots & \vdots & \vdots & \vdots & \ddots & \vdots & \vdots \\
0 & 0 & 0 & 0 & \cdots & 0 & 1 \\
0 & 0 & 0 & 0 & \cdots & 0 & 0
\end{array}\right], \\
B & =\left[\begin{array}{c}
0 \\
0 \\
\vdots \\
0 \\
1
\end{array}\right]
\end{aligned}
$$

$$
C=\left[\begin{array}{c}
1 \\
0 \\
\vdots \\
0 \\
0
\end{array}\right] .
$$

Take a strict Hurwitz matrix $\bar{A}=A-B k_{0}^{T}$. So, there is a positive definite symmetry matrix, $P, \bar{P}$ meets Lyapunov equation $\Lambda^{T} P+P \Lambda=-Q$, and $\bar{A}^{T} \bar{P}+\bar{P} \bar{A}=-\bar{Q}$, where $\bar{Q}$ and $Q$ are $n \times n$ d-order positive definite symmetric matrices. Take $V_{\bar{e}}=(1 / 2) e^{-T} P \bar{e}$ because $\bar{Q}$ and the design of $k_{c}$ are determined by the designer. We can choose $\bar{Q}$ and $k_{c}$, and satisfy $\dot{V}_{\bar{e}} \leq 0$. Therefore, $V_{\bar{e}}$ is a bounded function and has a constant value that satisfies $\mathrm{v}, \bar{V}_{\bar{e}}$, and meets $V_{\bar{e}} \leq \bar{V}_{\bar{e}}$.

$\mathrm{We}$ are going to design a hybrid adaptive control and make the closed-loop system output $y(t)$ trace $y_{m}(t)$. We use $\hat{f}(\bar{X}), \hat{g}(\bar{X})$, and $u_{d}(\bar{X})$ to replace them separately, $\widehat{f}\left(\bar{X} \mid \theta_{f}\right), \widehat{g}\left(\bar{X} \mid \theta_{g}\right)$, and $u_{D}\left(\bar{X} \mid \theta_{D}\right)$. Therefore, error dynamic equation (29) can be written as follows:

$$
\begin{aligned}
\dot{\tilde{e}}= & A \widetilde{e}-B \alpha\left[\hat{f}\left(\bar{X} \mid \theta_{f}\right)-f(X)+\hat{g}\left(\bar{X} \mid \theta_{g}\right)-g(X) u_{I}\right] \\
& +(1-\alpha) g(X)\left(u^{*}-u_{D}\left(\bar{X} \mid \theta_{D}\right)\right)+B g(X)\left(u_{s}+u_{c}\right)-B d,
\end{aligned}
$$

and taking $V_{\widetilde{e}}=(1 / 2) \widetilde{e}^{T} P \widetilde{e}$ and using (31), we can get

$$
\begin{aligned}
\dot{V}_{\widetilde{e}} \leq & -\frac{1}{2} \widetilde{e}^{T} Q \widetilde{e}+\left|\widetilde{e}^{T} P B\right| \alpha\left[\left|\widehat{f}\left(\bar{X} \mid \theta_{f}\right)\right|-|f(X)|\right. \\
& \left.+\left|\hat{g}\left(\bar{X} \mid \theta_{g}\right) u_{I}\right|+\left|g(X) u_{I}\right|\right]+\left|(1-\alpha) g(X) u^{*}\right| \\
& \left.+\mid g(X) u_{D}\left(\bar{X} \mid \theta_{D}\right)\right)|+| \widetilde{e}^{T} P B \mid\left[(1-\alpha)\left|g(X) \alpha+u_{c}\right|\right] \\
& -\tilde{e}^{T} P B g(X) u_{s} .
\end{aligned}
$$

In order to design $u_{s}$ to satisfy $\widetilde{V}_{\bar{e}} \leq 0$, choose supervisory control $u_{s}$ as follows:

$$
\begin{aligned}
u_{s}= & \left(\frac{V_{\widetilde{e}}}{\bar{V}}\right)^{p} \operatorname{sgn}\left(\tilde{e}^{T} P B\right)\left[\frac{\alpha}{g_{l}(X)}\left|\widehat{f}\left(\bar{X} \mid \theta_{f}\right)\right|+f^{u}\left(\left|\hat{g}\left(\bar{X} \mid \theta_{g}\right)\right|+g^{u}\left|u_{I}\right|\right)\right. \\
& \left.+\left(f^{u}(X)+\left|y_{m}^{(n)}\right|+\left|k^{T} e\right|\right)\right]++\left(\frac{V_{\tilde{e}}}{\bar{V}}\right)^{p} \operatorname{sgn}\left(\widetilde{e}^{T} P B\right)(1-\alpha)\left(\left[\left|u_{d}\right|\right]\right. \\
& \left.+\frac{1}{g_{l}}\left(f^{u}(X)+\left|y_{m}^{(n)}\right|+\left|k_{0}^{T} \bar{e}\right|\right)+g^{u}\left|u_{c}\right|+d_{m}\right) .
\end{aligned}
$$

We also chose $u_{c}=c_{0} \operatorname{sgn}\left(\widetilde{e}^{T} P B\right)$, where $c_{0}$ is a nonnegative real number. Taking into account the situation $V_{\bar{e}}>\bar{V}$ and bringing (33) into (32), we can get $\dot{V}_{\bar{e}} \leq-(1 / 2) e^{-T} Q \widetilde{e} \leq 0$. So, the fuzzy controller $u$ of the closed-loop system is

$$
\begin{aligned}
u= & \alpha \widehat{g}^{-1}\left(\bar{X} \mid \theta_{g}\right)\left[-\hat{f}\left(\bar{X} \mid \theta_{f}\right)+\left|y_{n}^{m}\right|+\left|k_{0}^{T} \bar{e}\right|\right] \\
& +(1-\alpha) u_{D}\left(\bar{X} \mid \theta_{D}\right) u_{s}+u_{c} .
\end{aligned}
$$

It is not a big mistake to get the error in our work $V_{\bar{e}} \leq \bar{V}$; then, the supervisory controller $u_{s}$ is zero at this time. 
8

Mathematical Problems in Engineering

If the system tends to separate, for example, $V_{\bar{e}}>\bar{V}$, then we use $u_{s}$ to force $V_{\bar{e}} \leq \bar{V}$.

4. Design of the Adaptive Rule

In the fuzzy logic system, in order to adjust the parameters, therefore, the optimal parameters $\theta_{f l}^{*}, \theta_{f r}^{*}, \theta_{g r}^{*}, \theta_{g l}^{*}, \theta_{g}^{*}, \theta_{D l}^{*}$, and $\theta_{D r}^{*}$ are defined as follows:

$$
\begin{aligned}
& \theta_{f l}^{*}=\arg \min _{\theta_{f l} \in \Omega_{f}}\left[\sup _{X \in \Omega_{x}, \bar{X} \in \Omega_{\bar{x}}}\left|\widehat{f}_{l}\left(\bar{X} / \theta_{f l}\right)-f(X)\right|\right], \\
& \theta_{f r}^{*}=\arg \min _{\theta_{f r} \in \Omega_{f}}\left[\sup _{X \in \Omega_{x}, \bar{X} \in \Omega_{\bar{x}}}\left|\widehat{f}_{r}\left(\bar{X} / \theta_{f r}\right)-f(X)\right|\right], \\
& \theta_{g l}^{*}=\arg \min _{\theta_{g l} \in \Omega_{g}}\left[\sup _{X \in \Omega_{x}, \bar{X} \in \Omega_{\bar{x}}}\left|\hat{g}_{l}\left(\bar{X} / \theta_{g l}\right)-g(X)\right|\right], \\
& \theta_{g r}^{*}=\arg \min _{\theta_{g r} \in \Omega_{g}}\left[\sup _{X \in \Omega_{x}, \bar{X} \in \Omega_{\bar{x}}}\left|\widehat{g}_{r}\left(\bar{X} / \theta_{g r}\right)-g(X)\right|\right], \\
& \theta_{D l}^{*}=\arg \min _{\theta_{D l} \in \Omega_{D}}\left[\sup _{X \in \Omega_{x}, \bar{X} \in \Omega_{\bar{x}}}\left|u^{*}(X)-u_{l}\left(\bar{X} / \theta_{D l}\right)\right|\right], \\
& \theta_{D r}^{*}=\arg \min _{\theta_{D r} \in \Omega_{D}}\left[\sup _{X \in \Omega_{x}, \bar{X} \in \Omega_{\bar{x}}}\left|u^{*}(X)-u_{r}\left(\bar{X} / \theta_{D r}\right)\right|\right] .
\end{aligned}
$$

Here, $\Omega_{f}, \Omega_{g}, \Omega_{D}, \Omega_{X}$, and $\Omega_{\bar{X}}$ are the number of tight sets at the boundary $\theta_{f l}^{*}, \theta_{f r}^{*}, \theta_{g r}^{*}, \theta_{g l}^{*}$, and $\theta_{D l}^{*}, \theta_{D r}^{*}$, and $X$ and $\bar{X}$ are defined as

$$
\begin{aligned}
& \Omega_{f}=\left\{\theta_{f l}^{i}, \theta_{f r}^{i}:\left\|\theta_{f l}^{i}\right\| \leq M_{f},\left\|\theta_{f r}^{i}\right\| \leq M_{f}\right\}, \\
& \Omega_{g}=\left\{\theta_{g l}^{i}, \theta_{g r}^{i}:\left\|\theta_{g l}^{i}\right\| \leq M_{g},\left\|\theta_{g r}^{i}\right\| \leq M_{g}\right\}, \\
& \Omega_{D}=\left\{\theta_{D l}^{i}, \theta_{D r}^{i}:\left\|\theta_{D l}^{i}\right\| \leq M_{D},\left\|\theta_{D r}^{i}\right\| \leq M_{D}\right\}, \\
& \Omega_{X}=\left\{X:\|X\| \leq M_{X}\right\}, \\
& \Omega_{\bar{X}}=\left\{\bar{X}:\|\bar{X}\| \leq M_{\bar{X}}\right\} .
\end{aligned}
$$

Here, $M_{f}, M_{g}, M_{D}, M_{X}$, and $M_{\bar{X}}$ are normal numbers. The minimum approximation error is defined as follows:

$$
\begin{aligned}
\omega= & \alpha\left[\hat{f}\left(\bar{X} \mid \theta_{f}^{*}\right)-f(X)+\hat{g}\left(\bar{X} \mid \theta_{g}^{*}\right)-g(X) u_{I}\right] \\
& +(1-\alpha) g(X)\left(u^{*}(X)-u\left(\bar{X} \mid \theta_{D}^{*}\right)\right)-d(t), \\
\dot{\tilde{e}}= & \Lambda \widetilde{e}-B g(X) u_{s}+B\left\{\alpha \left[\widehat{f}\left(X / \theta_{f}\right)-\widehat{f}\left(X / \theta_{f}^{*}\right)\right.\right. \\
& \left.\left.+\left(\hat{g}\left(X / \theta_{g}\right)-\hat{g}\left(X / \theta_{g}^{*}\right)\right) u_{I}\right]\right\} \\
& -B\left\{(1-\alpha) g(X)\left(u_{D}\left(X / \theta_{D}\right)-u_{D}\left(X / \theta_{D}^{*}\right)\right)+\omega\right\}
\end{aligned}
$$

$$
\begin{aligned}
\hat{f}\left(X / \theta_{f}\right)-\hat{f}\left(X / \theta_{f}^{*}\right) & \\
= & \frac{1}{2}\left(\sum_{i=1}^{L} q_{f l}^{i}\left(\theta_{f l}^{i}-\theta_{f l}^{i *}\right)+\sum_{j=L+1}^{M} q_{f l}^{j}\left(\theta_{f l}^{j}-\theta_{f l}^{j *}\right)\right) \vec{X} \\
& +\frac{1}{2}\left(\sum_{i=1}^{R} q_{f r}^{i}\left(\theta_{f r}^{i}-\theta_{f r}^{i *}\right)+\sum_{j=R+1}^{M} q_{f r}^{j}\left(\theta_{f r}^{j}-\theta_{f r}^{j *}\right)\right) \vec{X} \\
= & \frac{1}{2}\left(\sum_{i=1}^{L} q_{f l}^{i} \Theta_{f l}^{i}+\sum_{j=L+1}^{M} q_{f l}^{j} \Theta_{f l}^{j}+\sum_{i=1}^{R} q_{f r}^{i} \Theta_{f r}^{i}+\sum_{j=R+1}^{M} q_{f r}^{j} \Theta_{f r}^{j}\right) \vec{X}, \\
\Theta_{f l}^{i}= & \theta_{f r}^{i}-\theta_{f r}^{i *}, \\
\Theta_{f l}^{j}= & \theta_{f l}^{j}-\theta_{f l}^{j *}, \\
\Theta_{f r}^{j}= & \theta_{f r}^{j}-\theta_{f r}^{j *}, \\
\Theta_{f r}^{i}= & \theta_{f r}^{i}-\theta_{f r}^{i *},
\end{aligned}
$$

the same reason,

$$
\begin{aligned}
& \hat{g}\left(X / \theta_{g}\right)-\hat{g}\left(X / \theta_{g}^{*}\right) \\
& \quad=\frac{1}{2}\left(\sum_{i=1}^{L^{1}} q_{g l}^{i} \Theta_{g l}^{i}+\sum_{j=L^{1}+1}^{M} q_{g l}^{j} \Theta_{g l}^{j}+\sum_{i=1}^{R^{1}} q_{g r}^{i} \Theta_{g r}^{i}+\sum_{j=R^{1}+1}^{M} q_{g r}^{j} \Theta_{g r}^{j}\right) \vec{X} \\
& \widehat{u}\left(X / \theta_{u}\right)-\widehat{u}\left(X / \theta_{u}^{*}\right) \\
& \quad=\frac{1}{2}\left(\sum_{i=1}^{L^{2}} q_{u l}^{i} \Theta_{u l}^{i}+\sum_{j=L^{2}+1}^{M} q_{u l}^{j} \Theta_{u l}^{j}+\sum_{i=1}^{R^{2}} q_{u r}^{i} \Theta_{u r}^{i}+\sum_{j=R^{2}+1}^{M} q_{u r}^{j} \Theta_{u r}^{j}\right) \vec{X} .
\end{aligned}
$$

Therefore, (37) is

$$
\begin{aligned}
\dot{\tilde{e}}= & \Lambda \tilde{e}-B g(X) u_{s} \\
& +\frac{1}{2} B\left\{\alpha \left[\left(\sum_{i=1}^{L} q_{f l}^{i} \Theta_{f l}^{i}+\sum_{j=L+1}^{M} q_{f l}^{j} \Theta_{f l}^{j}+\sum_{i=1}^{R} q_{f r}^{i} \Theta_{f r}^{i}\right.\right.\right. \\
& \left.+\sum_{j=R+1}^{M} q_{f r}^{j} \Theta_{f r}^{j}\right) \\
& +\left(\sum_{i=1}^{L^{1}} q_{g l}^{i} \Theta_{g l}^{i}+\sum_{j=L^{1}+1}^{M} q_{g l}^{j} \Theta_{g l}^{j}+\sum_{i=1}^{R^{1}} q_{g r}^{i} \Theta_{g r}^{i}+\sum_{j=R^{1}+1}^{M} q_{g r}^{j} \Theta_{g r}^{j}\right) \vec{X}- \\
B\{ & (1-\alpha) g(X)\left(\frac { 1 } { 2 } \left(\sum_{i=1}^{L} q_{f l}^{i} \Theta_{f l}^{i}+\sum_{j=L+1}^{M} q_{f l}^{j} \Theta_{f l}^{j}\right.\right. \\
& \left.\left.\left.+\sum_{i=1}^{R} q_{f r}^{i} \Theta_{f r}^{i}+\sum_{j=R+1}^{M} q_{f r}^{j} \Theta_{f r}^{j}\right)\right)+\omega\right\} .
\end{aligned}
$$


Now consider the Lyapunov function:

$$
\begin{aligned}
V= & -\frac{1}{2} \widetilde{e}^{T} p \widetilde{e}+\frac{1}{2 \Gamma_{f}}\left(\theta_{f}-\theta_{f}^{*}\right)^{T}\left(\theta_{f}-\theta_{f}^{*}\right) \\
& +\frac{\alpha}{2 \Gamma_{g}}\left(\theta_{g}-\theta_{g}^{*}\right)^{T}\left(\theta_{g}-\theta_{g}^{*}\right)+\frac{(1-\alpha)}{2 \Gamma_{g}}\left(\theta_{D}-\theta_{D}^{*}\right)^{T}\left(\theta_{D}-\theta_{D}^{*}\right) .
\end{aligned}
$$
get

Bring (37) and (40) into (41), calculate and derive, and

$$
\begin{aligned}
& \dot{V}=-\frac{1}{2} \widetilde{e}^{T} Q e-\widetilde{e}^{T} P B g(X) u_{s}+\widetilde{e}^{T} P B \omega \\
& +\alpha\left[\sum_{i=1}^{L} \Theta_{f l}^{i}\left(\Gamma_{f l}^{i}\right)^{-1}\left(\left(\dot{\theta}_{f l}^{i}\right)^{T}+\frac{1}{2} \Gamma_{f l}^{i} B^{T} P \widetilde{e} q_{f l}^{i} \vec{X}\right)\right] \\
& +\alpha\left[\sum_{j=L+1}^{L} \Theta_{f l}^{j}\left(\Gamma_{f l}^{j}\right)^{-1}\left(\left(\dot{\theta}_{f l}^{j}\right)^{T}+\frac{1}{2} \Gamma_{f l}^{j} B^{T} P \widetilde{e} q_{f l}^{j} \vec{X}\right)\right] \\
& +\alpha\left[\sum_{i=1}^{R} \Theta_{f r}^{i}\left(\Gamma_{f r}^{i}\right)^{-1}\left(\left(\dot{\theta}_{f r}^{i}\right)^{T}+\frac{1}{2} \Gamma_{f r}^{i} B^{T} P \widetilde{e} q_{f r}^{i} \vec{X}\right)\right] \\
& +\alpha\left[\sum_{j=R+1}^{M} \Theta_{f r}^{j}\left(\Gamma_{f r}^{j}\right)^{-1}\left(\left(\dot{\theta}_{f r}^{j}\right)^{T}+\frac{1}{2} \Gamma_{f r}^{j} B^{T} P \widetilde{e} q_{f r}^{j} \vec{X}\right)\right] \\
& +\alpha\left[\sum_{i=1}^{L^{1}} \Theta_{g l}^{i}\left(\Gamma_{g l}^{i}\right)^{-1}\left(\left(\dot{\theta}_{g l}^{i}\right)^{T}+\frac{1}{2} \Gamma_{g l}^{i} B^{T} P \widetilde{e} q_{g l}^{i} \vec{X} u_{I}\right)\right] \\
& +\alpha\left[\sum_{j=L^{1}+1}^{M} \Theta_{g l}^{j}\left(\Gamma_{g l}^{j}\right)^{-1}\left(\left(\dot{\theta}_{g l}^{j}\right)^{T}+\frac{1}{2} \Gamma_{g l}^{j} B^{T} P \widetilde{e} q_{g l}^{j} \vec{X} u_{I}\right)\right] \\
& +\alpha\left[\sum_{i=R^{1}}^{R^{1}} \Theta_{g r}^{i}\left(\Gamma_{g r}^{i}\right)^{-1}\left(\left(\dot{\theta}_{g r}^{i}\right)^{T}+\frac{1}{2} \Gamma_{g r}^{i} B^{T} P \widetilde{e} q_{g r}^{i} \vec{X} u_{I}\right)\right] \\
& +\alpha\left[\sum_{j=R^{1}+1}^{M} \Theta_{g r}^{j}\left(\Gamma_{g r}^{j}\right)^{-1}\left(\left(\dot{\theta}_{g r}^{j}\right)^{T}+\frac{1}{2} \Gamma_{g r}^{j} B^{T} P \widetilde{e} q_{g r}^{j} \vec{X} u_{I}\right)\right] \\
& +(1-\alpha)\left[\sum_{i=1}^{R^{2}} \Theta_{u l}^{i}\left(\Gamma_{u l}^{i}\right)^{-1}\left(\left(\dot{\theta}_{u l}^{i}\right)^{T}+\frac{1}{2} \Gamma_{u l}^{i} B^{T} P \widetilde{e} q_{u l}^{i} \vec{X}\right)\right] \\
& +(1-\alpha)\left[\sum_{j=R^{2}+1}^{M} \Theta_{u l}^{j}\left(\Gamma_{u l}^{j}\right)^{-1}\left(\left(\dot{\theta}_{u l}^{j}\right)^{T}+\frac{1}{2} \Gamma_{u l}^{j} B^{T} P \widetilde{e} q_{u l}^{j} \vec{X}\right)\right] \\
& +(1-\alpha)\left[\sum_{i=1}^{R^{2}} \Theta_{u r}^{i}\left(\Gamma_{u r}^{i}\right)^{-1}\left(\left(\dot{\theta}_{u r}^{i}\right)^{T}+\frac{1}{2} \Gamma_{u r}^{i} B^{T} P \widetilde{e} q_{u r}^{i} \vec{X}\right)\right] \\
& +(1-\alpha)\left[\sum_{j=R^{2}+1}^{M} \Theta_{u r}^{j}\left(\Gamma_{u r}^{j}\right)^{-1}\left(\left(\dot{\theta}_{u r}^{j}\right)^{T}+\frac{1}{2} \Gamma_{u r}^{j} B^{T} P \widetilde{e} q_{u r}^{j} \vec{X}\right)\right], \\
& \Gamma_{f}=\left[\begin{array}{ll}
\Gamma_{f l} & \Gamma_{f r}
\end{array}\right], \\
& \Gamma_{f l}=\left[\begin{array}{ccc}
\tau_{f l 0} & & \\
& \ddots & \\
& & \tau_{f l n}
\end{array}\right] .
\end{aligned}
$$

$\Gamma_{g}$ and $\Gamma_{D}$ can be expressed similarly, and choose the following adaptive rules:

$$
\begin{aligned}
& \dot{\theta}_{f l}^{i}=-\frac{1}{2} \Gamma_{f l}^{i} B^{T} P \widetilde{e} q_{f l}^{i} \vec{X}, \\
& \dot{\theta}_{f l}^{j}=-\frac{1}{2} \Gamma_{f l}^{j} B^{T} P \widetilde{e} q_{f l}^{j} \vec{X},
\end{aligned}
$$$$
\dot{\theta}_{f r}^{j}=-\frac{1}{2} \Gamma_{f r}^{j} B^{T} P \widetilde{e} q_{f r}^{j} \vec{X}
$$$$
\dot{\theta}_{f r}^{i}=-\frac{1}{2} \Gamma_{f r}^{i} B^{T} P \widetilde{e} q_{f r}^{i} \vec{X}
$$$$
\dot{\theta}_{g l}^{i}=-\frac{1}{2} \Gamma_{g l}^{i} B^{T} P \widetilde{e} q_{g l}^{i} \vec{X} u_{I}
$$$$
\dot{\theta}_{g l}^{j}=-\frac{1}{2} \Gamma_{g l}^{j} B^{T} P \widetilde{e} q_{g l}^{j} \vec{X} u_{I}
$$$$
\dot{\theta}_{g r}^{j}=-\frac{1}{2} \Gamma_{g r}^{j} B^{T} P \widetilde{e} q_{g r}^{j} \vec{X} u_{I},
$$$$
\dot{\theta}_{g r}^{i}=-\frac{1}{2} \Gamma_{g r}^{i} B^{T} P \widetilde{e} q_{g r}^{i} \vec{X} u_{I},
$$$$
\dot{\theta}_{u l}^{i}=-\frac{1}{2} \Gamma_{u l}^{i} B^{T} P \widetilde{e} q_{u l}^{i} \vec{X}
$$$$
\dot{\theta}_{u r}^{i}=-\frac{1}{2} \Gamma_{u r}^{i} B^{T} P \widetilde{e} q_{u r}^{i} \vec{X},
$$$$
\dot{\theta}_{u r}^{j}=-\frac{1}{2} \Gamma_{u r}^{j} B^{T} P \widetilde{e} q_{u r}^{j} \vec{X},
$$$$
\dot{\theta}_{u l}^{j}=-\frac{1}{2} \Gamma_{u l}^{j} B^{T} P \widetilde{e} q_{u l}^{j} \vec{X} .
$$

So,

$$
\begin{gathered}
\left(\dot{\theta}_{f l}^{k}\right)^{T}=\left(\begin{array}{c}
a_{f l 0}^{k} \\
\vdots \\
a_{f l n}^{k}
\end{array}\right)=-\frac{1}{2} B^{T} P \widetilde{e} q_{f l}^{i}\left(\begin{array}{c}
\tau_{f l 0}^{k} \\
\tau_{f l 1}^{k} x_{1} \\
\vdots \\
\tau_{f l n}^{k} x_{n}
\end{array}\right), \\
\left(\dot{\theta}_{f r}^{k}\right)^{T}=\left(\begin{array}{c}
a_{f r 0}^{k} \\
\vdots \\
\tau_{f r 0}^{k} \\
a_{f r n}^{k}
\end{array}\right)=-\frac{1}{2} B^{T} P \widetilde{e} q_{f r}^{i}\left(\begin{array}{c}
\tau_{f r 1}^{k} x_{1} \\
\vdots \\
\tau_{f r n}^{k} x_{n}
\end{array}\right),
\end{gathered}
$$

$k=1,2, \cdots, M$ 


$$
\begin{aligned}
& a_{f l 0}^{k}=-\frac{1}{2} \tau_{f l 0}^{k} B^{T} P \widetilde{e} q_{f l}^{i}, \\
& a_{f l k}^{k}=-\frac{1}{2} \tau_{f l k}^{k} B^{T} P \widetilde{e} q_{f l}^{i} x_{k}, k=1,2, \cdots, n, \\
& a_{f r 0}^{k}=-\frac{1}{2} \tau_{f r 0}^{k} B^{T} P \widetilde{e} q_{f r}^{i}, \\
& a_{f r k}^{k}=-\frac{1}{2} \tau_{f r k}^{k} B^{T} P \widetilde{e} q_{f r}^{i} x_{k}, \\
& b_{g r 0}^{k}=-\frac{1}{2} \tau_{g r 0}^{k} B^{T} P \widetilde{e} q_{g r}^{i} u_{I}, \\
& b_{g r k}^{k}=-\frac{1}{2} \tau_{g r k}^{k} B^{T} P \widetilde{e} q_{g r}^{i} x_{k} u_{I}, \\
& b_{g l 0}^{k}=-\frac{1}{2} \tau_{g l 0}^{k} B^{T} P \widetilde{e} q_{g l}^{i} u_{I}, \\
& b_{g l k}^{k}=-\frac{1}{2} \tau_{g l k}^{k} B^{T} P \widetilde{e} q_{g l}^{i} x_{k} u_{I}, \\
& u_{u l 0}^{k}=-\frac{1}{2} \tau_{u l 0}^{k} B^{T} P \widetilde{e} q_{u l}^{i}, \\
& u_{u r 0}^{k}=-\frac{1}{2} \tau_{u r 0}^{k} B^{T} P \widetilde{e} q_{u r}^{i}, \\
& u_{u l k}^{k}=-\frac{1}{2} \tau_{u l k}^{k} B^{T} P \widetilde{e} q_{u l}^{i} x_{k}, \\
& \frac{1}{2} \tau_{u r k}^{k} B^{T} P \widetilde{e} q_{u r}^{i} x_{k} . \\
& u_{u r}, \\
& u^{k},
\end{aligned}
$$$$
\left\|\theta_{u l}^{k}\right\|=M_{u}
$$$$
\left\|\theta_{u l}^{k}\right\| \leq M_{u}
$$

And (42) is

$$
\dot{V}=-\frac{1}{2} \widetilde{e}^{T} Q e-\tilde{e}^{T} P B g(X) u_{s}+\tilde{e}^{T} P B \omega
$$

After bringing in (31),

$$
\dot{V} \leq-\frac{1}{2} \widetilde{e}^{T} Q e+\widetilde{e}^{T} P B \omega .
$$

\section{Stability Analysis}

Theorem 1. All parameter variables $\left\|\theta_{u l}^{k}\right\|<M_{u},\left\|\theta_{u r}^{k}\right\|<M_{u}$, $\left\|\theta_{f r}^{k}\right\|<M_{f},\left\|\theta_{f l}^{k}\right\|<M_{f},\left\|\theta_{g l}^{k}\right\|<M_{g}$, and $\left\|\theta_{g r}^{k}\right\|<M_{g}$ are bounded, $k=1,2, \cdots, M$.

Proof. Suppose

$$
V_{u l}^{k}=\frac{1}{2}\left(\theta_{u l}^{k}\right)^{T} \theta_{u l}^{k} .
$$

So,

$$
\dot{V}_{u l}^{k}=\frac{1}{2}\left(\dot{\theta}_{u l}^{k}\right)^{T} \theta_{u l}^{k}+\frac{1}{2}\left(\theta_{u l}^{k}\right)^{T} \dot{\theta}_{u l}^{k}
$$

if

$$
\left(\dot{\theta}_{u l}^{k}\right)^{T}=-\frac{1}{2} \Gamma_{u l}^{i} B^{T} P \widetilde{e} q_{u l}^{i} \vec{X}
$$

when 
Here, $x_{1}(t)$ means the total number of food at the time of $\mathrm{t}, x_{2}(t)$ is the total number of predators, $a, b, c, e_{d}, \gamma, \alpha, \beta$ are the regular constant ecology, $\gamma$ is the transforming factor, $e_{d}$ represents the death ratio of predators, $\bar{h}(x)$ is a function of Holling's functional responses, and $x_{2}\left(\alpha x^{2} /\left(1+\beta x^{2}\right)\right)$ is the third kind of Holling's functional responses. In order to reach an ideal ecology balance in this two-dimension predator system, we get a way to control it:

$$
\begin{aligned}
& \bar{h}(x)=0.0012 x_{1}^{3}, \\
& a=1.0, \\
& b=0.0014, \\
& c=0.06, \\
& e_{d}=0.2, \\
& \gamma=0.08, \\
& \alpha=5.0, \\
& \beta=1.2 . \\
& \text { We get } \\
& \frac{d x_{1}}{d t}=x_{1}\left[\left(1+1.2 x_{1}^{2}\right)(1-\right.\left.\left.0.06 x_{1}-0.0014 x_{1}^{2}-0.0012 x_{1}^{3}\right)-x_{1} x_{2}\right], \\
& \frac{d x_{2}}{d t}=x_{2}\left(0.16 x_{1}^{2}-0.2\right)+u \\
& y=x_{1} .
\end{aligned}
$$

In order to establish the direct relation between output $y$ and controller $u$, we need to derive $y$. After derivating twice, we get

$$
\begin{aligned}
\ddot{y}= & \ddot{x}_{1} \\
= & x_{1}\left[\left(1+1.2 x_{1}^{2}\right)\left(0.8-0.06 x_{1}-0.0014 x_{1}^{2}-0.0012 x_{1}^{3}\right)-x_{1} x_{2}\right]^{2} \\
& -x_{1}^{2} x_{2}\left(0.8-0.06 x_{1}+1.3586 x_{1}^{2}-0.0732 x_{1}^{3}-0.00168 x_{1}^{4}\right. \\
& \left.-0.00144 x_{1}^{5}-x_{1} x_{2}\right)+2.3972 x_{1}^{2}-0.2196 x_{1}^{3}-0.00672 x_{1}^{4} \\
& -0.0072 x_{1}^{5}-0.06 x_{1}-x_{1}^{2} u .
\end{aligned}
$$

Here, we command

$$
\begin{aligned}
f(X) & \\
= & x_{1}\left[\left(1+1.2 x_{1}^{2}\right)\left(0.8-0.06 x_{1}-0.0014 x_{1}^{2}-0.0012 x_{1}^{3}\right)-x_{1} x_{2}\right]^{2} \\
& -x_{1}^{2} x_{2}\left(0.8-0.06 x_{1}+1.3586 x_{1}^{2}-0.0732 x_{1}^{3}-0.00168 x_{1}^{4}\right. \\
& \left.-0.00144 x_{1}^{5}-x_{1} x_{2}\right)+2.3972 x_{1}^{2}-0.2196 x_{1}^{3}-0.00672 x_{1}^{4} \\
& -0.0072 x_{1}^{5}-0.06 x_{1}, \\
b(X)= & -x_{1}^{2} .
\end{aligned}
$$

Fuzzy functions $\widehat{h}(x)$ and $\hat{g}(x)$ are based on the following rules:

$R_{h}$ : if $x_{1}$ is $A_{1}^{l}$ and $x_{2}$ is $A_{2}^{l}$, then

$$
\widehat{h}(X)=a_{0}^{l}=\frac{3}{2}-\vartheta\left[\frac{\lambda_{h}^{l}-\lambda}{\sigma_{h}^{l}+\sigma}\right](l=1, \cdots, m) .
$$

$R_{g}:$ if $x_{1}$ is $B_{1}^{l}$ and $x_{2}$ is $B_{2}^{l}$, then

$$
\widehat{g}(X)=b_{0}^{l}=\frac{3}{2}-\vartheta\left[\frac{\lambda_{g}^{l}-\lambda}{\sigma_{g}^{l}+\sigma}\right](l=1, \cdots, m),
$$

and from [25], we know $0.2<\left|x_{1}\right|<5.5,1.5<\left|x_{2}\right|<5$, and choose $\tau_{h 0}^{l}=50, \tau_{g 0}^{l}=1$. We choose $\varepsilon_{h}=0.5, \varepsilon_{g}=0.4$, $M_{0}(X)=7.7$, and $M_{1}(X)=3$.

Now, we request the target referring signal as $y_{m}=1.0$ and $|u| \leq 5$. The adaptive law comes out as the following:

$$
\begin{aligned}
& a_{f l 0}^{k}=-\frac{1}{2} \tau_{f l 0}^{k} B^{T} P \widetilde{e} q_{f l}^{i}, \\
& a_{f l k}^{k}=-\frac{1}{2} \tau_{f l k}^{k} B^{T} P \widetilde{e} q_{f l}^{i} x_{k}, k=1,2, \cdots, n, \\
& a_{f r 0}^{k}=-\frac{1}{2} \tau_{f r 0}^{k} B^{T} P \widetilde{e} q_{f r}^{i}, \\
& a_{f r k}^{k}=-\frac{1}{2} \tau_{f r k}^{k} B^{T} P \widetilde{e} q_{f r}^{i} x_{k} \\
& b_{g r 0}^{k}=-\frac{1}{2} \tau_{g r 0}^{k} B^{T} P \widetilde{e} q_{g r}^{i} u_{I}, \\
& b_{g r k}^{k}=-\frac{1}{2} \tau_{g r k}^{k} B^{T} P \widetilde{e} q_{g r}^{i} x_{k} u_{I}, \\
& b_{g l 0}^{k}=-\frac{1}{2} \tau_{g l 0}^{k} B^{T} P \widetilde{e} q_{g l}^{i} u_{I}, \\
& b_{g l k}^{k}=-\frac{1}{2} \tau_{g l k}^{k} B^{T} P \widetilde{e} q_{g l}^{i} x_{k} u_{I}, \\
& u_{u l 0}^{k}=-\frac{1}{2} \tau_{u l 0}^{k} B^{T} P \widetilde{e} q_{u l}^{i}, \\
& u_{u l k}^{k}=-\frac{1}{2} \tau_{u l k}^{k} B^{T} P \widetilde{e} q_{u l}^{i} x_{k} \\
& u_{u r 0}^{k}=-\frac{1}{2} \tau_{u r 0}^{k} B^{T} P \widetilde{e} q_{u r}^{i}, \\
& u_{u r k}^{k}=-\frac{1}{2} \tau_{u r k}^{k} B^{T} P \widetilde{e} q_{u r}^{i} x_{k} .
\end{aligned}
$$

From (55), (57), and (58) we get the controller: 


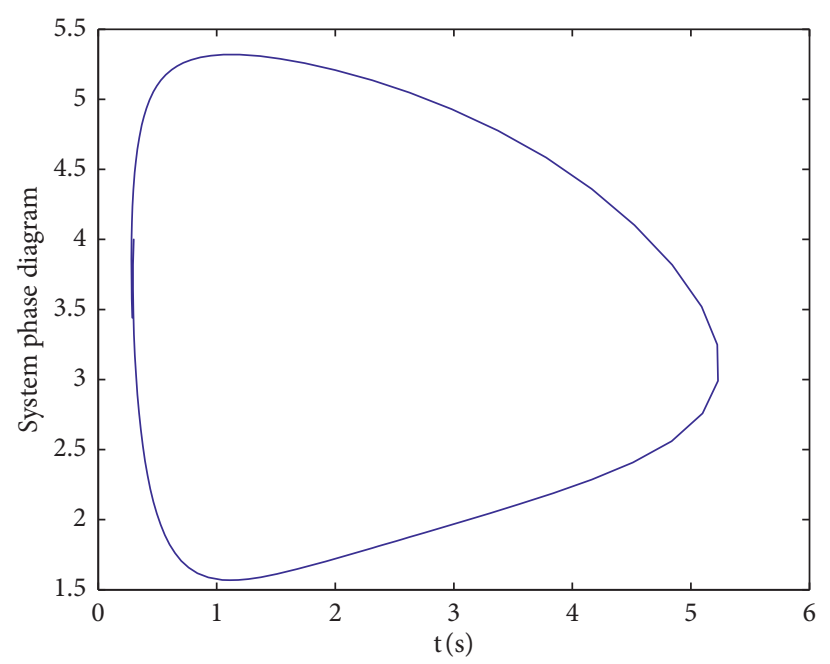

Figure 1: Two-dimensional system diagram.

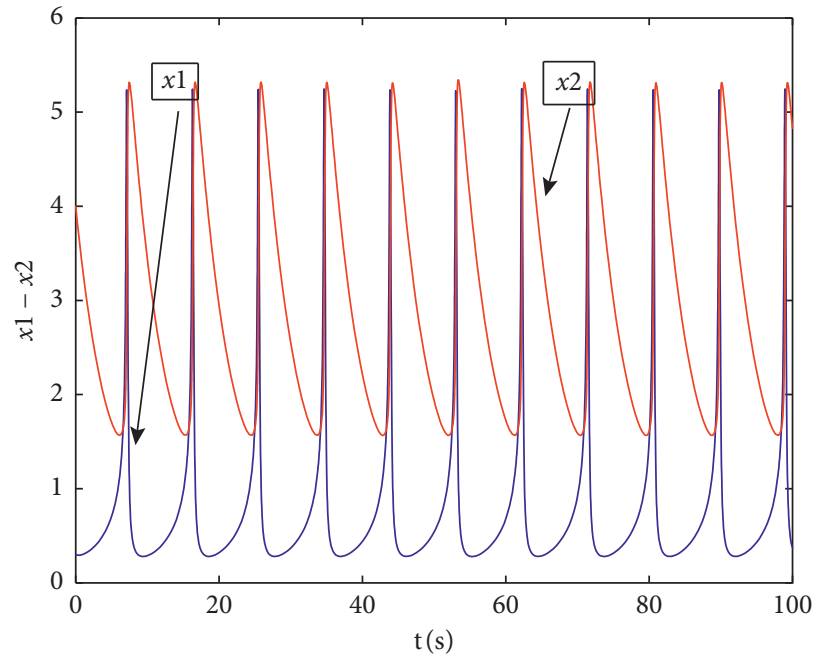

Figure 2: Two-dimensional system of fuzzy control.

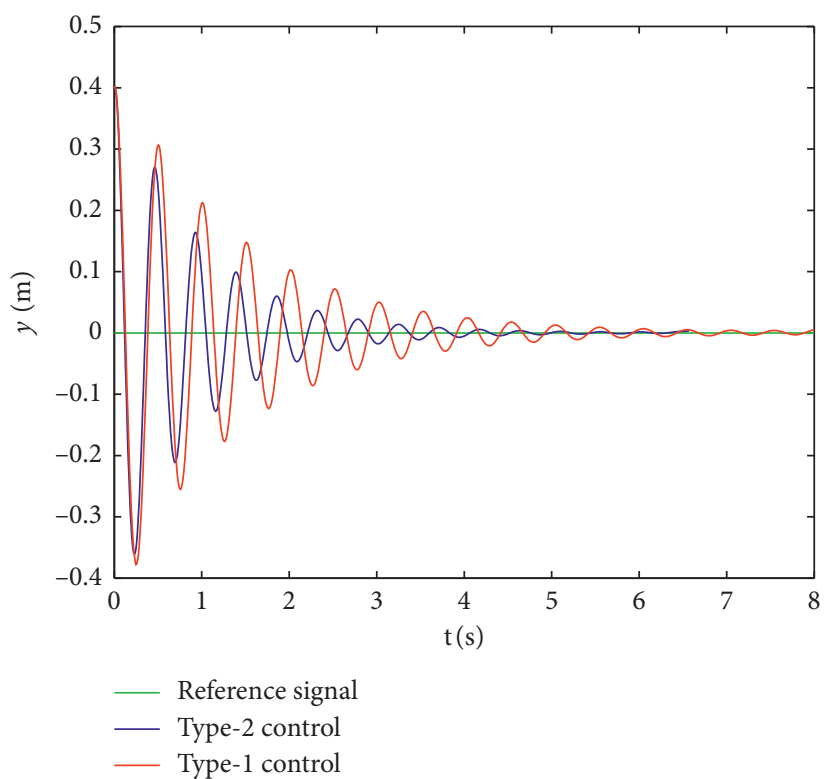

Figure 3: Control the comparison between type-1 and type-2. 


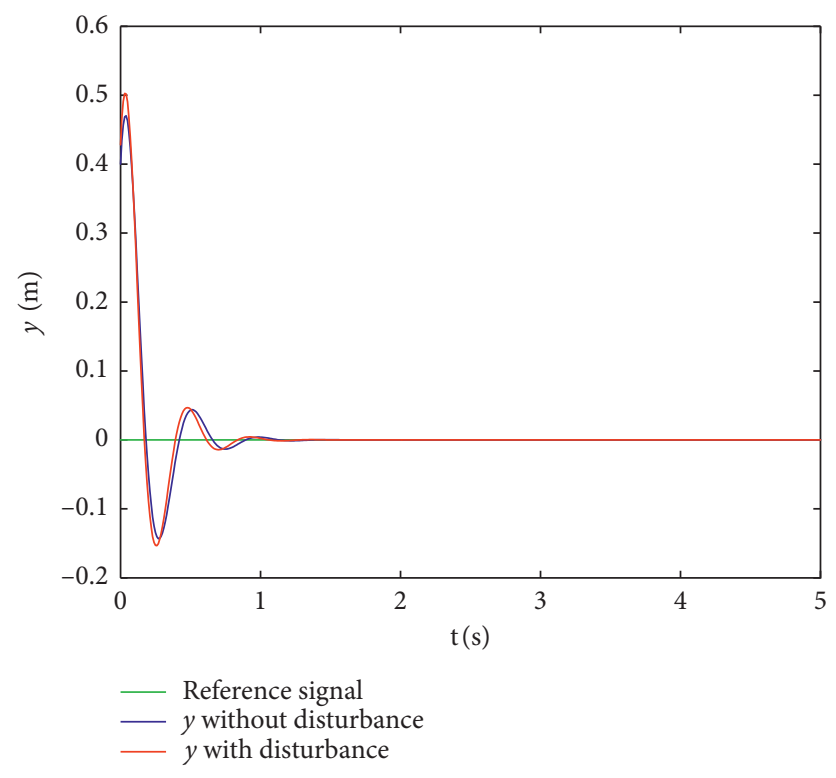

FIgURE 4: Comparison of $y$ output with incorrect perturbations.

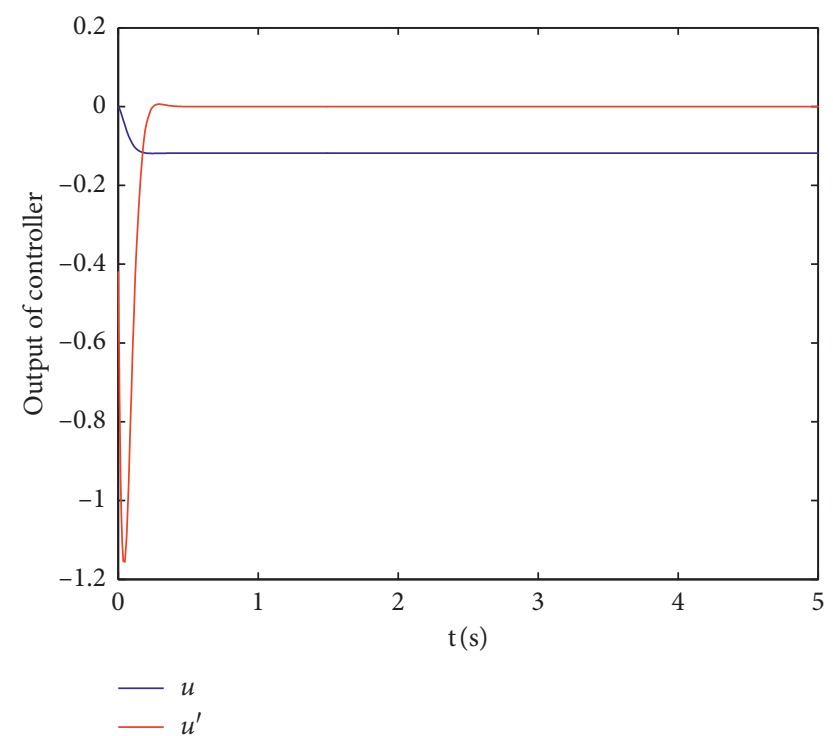

Figure 5: Fuzzy controller output curve.

$$
\begin{aligned}
u= & \frac{1}{x_{1}}\left[\left(1+1.2 x_{1}^{2}\right)\left(0.8-0.06 x_{1}-0.0014 x_{1}^{2}-0.0012 x_{1}^{3}\right)-x_{1} x_{2}\right]^{2} \\
& -x_{2}\left(0.8-0.06 x_{1}+1.3586 x_{1}^{2}-0.0732 x_{1}^{3}-0.00168 x_{1}^{4}-0.00144 x_{1}^{5}\right. \\
& \left.-x_{1} x_{2}-\frac{2}{x_{1}^{2}}\right)+2.3972-0.2196 x_{1}-0.00672 x_{1}^{2}-0.0072 x_{1}^{3} \\
& +\frac{0.94}{x_{1}}-\frac{1}{x_{1}^{2}}
\end{aligned}
$$

We choose $x_{1}(0)=0.3, x_{2}(0)=4.0, \varphi=0.1$, and $\phi=0.01$; then, simulation Figures $1-6$ are obtained.

It can be seen from Figures 1 and 2 that the variables of the controlled system are bounded, and the number of two variables (predator and prey) reaches a harmonious state. Figure 3 shows that the type- 2 fuzzy control has smaller fluctuation and better convergence. When $t=8$, the trajectory tends to zero, which indicates that the control system is 


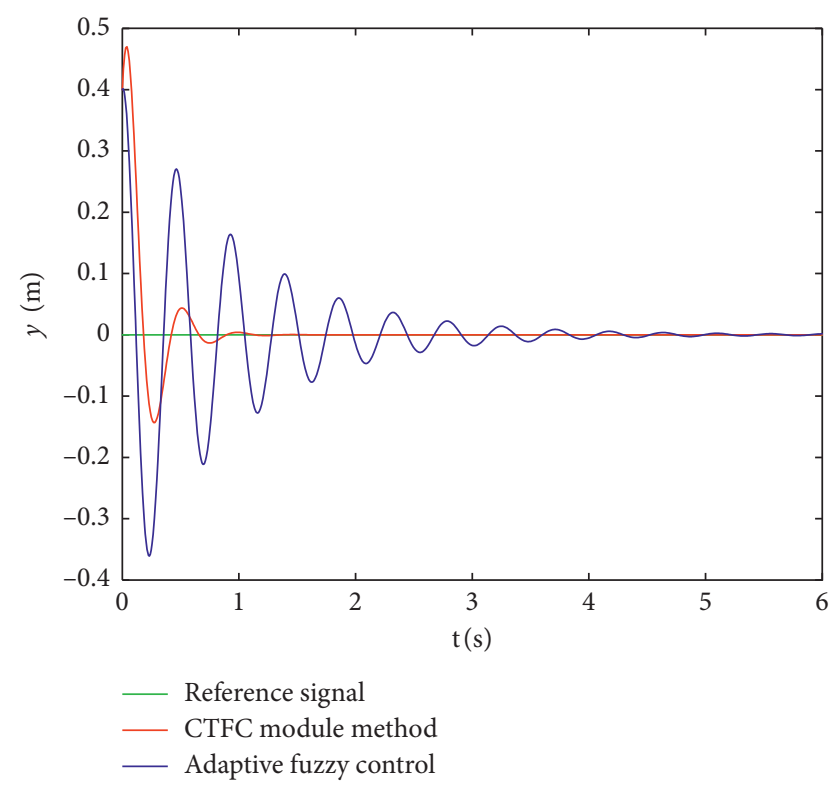

Figure 6: Control comparison diagram.

stable. Figures $4-6$ show that when the system is subject to the random interference, the output of the type- 2 combined T-S fuzzy control system can still achieve stability faster with less fluctuation than the type-2 traditional control system, which demonstrates the superiority of the proposed method.

\section{Conclusion}

This paper proposed a new design method of the interval type- 2 combined T-S adaptive fuzzy controller. By using Lyapunov's synthesis method, the global stability of the closed-loop system is studied under the meaning of uniformly bounded variables. The proposed method combines the nonlinear approximation ability, the local linearity of the dynamic fuzzy system, and the adaptive fuzzy control method of the linear system. Under this method, the design of the membership function does not require accurately, which can avoid the dependence of fuzzy adaptive control on membership function. The simulation results show that the proposed type- 2 T-S combined adaptive fuzzy controller is better than the type-1 T-S fuzzy adaptive control method. Besides, in dealing with unknown internal interference and error approximation, the type-2 fuzzy system needs to select fewer rules, and the membership function does not satisfy strict conditions compared with the type-1 method.

\section{Data Availability}

The data used to support the findings of this study are included within the article.

\section{Conflicts of Interest}

The authors declare that there are no conflicts of interest regarding the publication of this paper.

\section{Acknowledgments}

This work was supported by the National Key Research and Development Grant Program subtopic (2017YFC0804408), the National Natural Science Foundation of China (71573256), the Major Projects of National Social Science Foundation (16ZDA056), and 2019 Anhui Province Humanities and Social Science Key Research Project (SK2019A0540).

\section{References}

[1] X. Yin, M. Lei, and H. Pan, "Direct optimal power extraction control for a tidal turbine system based on fuzzy power tuning,” Ocean Engineering, vol. 170, pp. 426-433, 2018.

[2] P. Sarhad and B. Rezaie, "Adaptive predictive control based on adaptive neuro-fuzzy inference system for a class of nonlinear industrial processes," Journal of the Taiwan Institute of Chemical Engineers, vol. 61, pp. 132-137, 2018.

[3] E. H. Mamdani, "Advances in the linguistic synthesis of fuzzy controllers," International Journal of Man-Machine Studies, vol. 8, no. 6, pp. 669-678, 1976.

[4] Y. X. Jia, "Design of fuzzy control systems for greenhouse," Journal of the Hebei Academy of Sciences, vol. 23, pp. 42-44, 2006.

[5] T. Takagi and M. Sugeno, "Fuzzy identification of systems and its applications to modeling and control," IEEE Transactions on Systems, Man, and Cybernetics, vol. 15, no. 1, pp. 116-132, 1985.

[6] H. Ying, "General SISO Takagi-Sugeno fuzzy system with linear rule consequent are approximators," IEEE Transactions on Fuzzy Systems, vol. 6, pp. 582-587, 1998.

[7] E. H. Mamdani and S. Assilian, "An experiment in linguistic synthesis with a fuzzy logic controller," International Journal of Man-Machine Studies, vol. 7, no. 1, pp. 1-13, 1975.

[8] N. Wang, J.-C. Sun, and Y.-C. Liu, "Direct adaptive selfstructuring fuzzy control with interpretable fuzzy rules for a class of nonlinear uncertain systems," Neurocomputing, vol. 173, pp. 1640-1645, 2016.

[9] M.-R. Akbarzadeh, S. A. Hosseini, and M.-B. Naghibi-Sistani, "Stable indirect adaptive interval type-2 fuzzy sliding-based control and synchronization of two different chaotic systems," Applied Soft Computing, vol. 55, pp. 576-587, 2017.

[10] F. Baghbani, M.-R. Akbarzadeh, and A. Akbarzadeh, "Indirect adaptive robust mixed $\mathrm{H} 2 / \mathrm{H} \infty$ general type-2 fuzzy control of uncertain nonlinear systems," Applied Soft Computing, vol. 72, pp. 392-418, 2018.

[11] H. Sudheer, S. F. Kodad, and B. Sarvesh, "Improvements in direct torque control of induction motor for wide range of speed operation using fuzzy logic," Journal of Electrical Systems and Information Technology, vol. 5, no. 3, pp. 813-828, 2018.

[12] S. A. Hosseini, M. R. . Akbarzadeh, and M. B. Sistani, "A synchronizing controller using a direct adaptive interval type2 fuzzy sliding mode strategy," IEEE International Conference on Fuzzy Systems, vol. 446, pp. 1-8, 2013.

[13] L. A. Zadeh, "Fuzzy sets," Information and Control, vol. 8, no. 3, pp. 338-353, 1965.

[14] L. A. Zadeh, "The concept of a linguistic variable and its application to approximate reasoning," Information Sciences, vol. 2, no. 8,1975 .

[15] J. M. Mendel, "Advances in type-2 fuzzy sets and systems," Information Sciences, vol. 177, no. 1, pp. 84-110, 2007. 
[16] Z. Du, Y. Kao, and J. H. Park, "Interval type-2 fuzzy sampleddata control of time-delay systems," Information Sciences, vol. 487, pp. 193-207, 2019.

[17] Z. Du, Z. Yan, and Z. Zhao, "Interval type-2 fuzzy tracking control for nonlinear systems via sampled-data controller," Fuzzy Sets and Systems, vol. 356, pp. 92-112, 2019.

[18] T. Zhao, Z. Wei, S. Dian, and J. Xiao, "Observer-based Ho controller design for interval type-2 T-S fuzzy systems," Neurocomputing, vol. 177, pp. 9-25, 2016.

[19] J. Hua, L.-X. An, and Y.-M. Li, "Bionic fuzzy sliding mode control and robustness analysis," Applied Mathematical Modelling, vol. 39, no. 15, pp. 4482-4493, 2015.

[20] Y. M. L. Zhao, "Type-2 fuzzy mathematical modeling and analysis of the dynamical behaviors of complex ecosystems," Simulation Modelling Practice and Theory, vol. 16, pp. 13791391, 2008.

[21] W.-J. Chang and F.-L. Hsu, "Sliding mode fuzzy control for Takagi-Sugeno fuzzy systems with bilinear consequent part subject to multiple constraints," Information Sciences, vol. 327, pp. 258-271, 2016.

[22] C. Han, G. Zhang, L. Wu, and Q. Zeng, "Sliding mode control of T-S fuzzy descriptor systems with time-delay," Journal of the Franklin Institute, vol. 349, no. 4, pp. 1430-1444, 2012.

[23] B.-K. Xie and S.-J. Lee, "An extended type-reduction method for general type-2 fuzzy sets," IEEE Transactions on Fuzzy Systems, vol. 25, no. 3, pp. 715-724, 2017.

[24] F. Zhang, J. Hua, and Y. Li, "Indirect adaptive type-2 bionic fuzzy control," Applied Intelligence, vol. 48, no. 3, pp. 541-554, 2018.

[25] F. Zhang, Y. Li, and J. Hua, "Direct adaptive fuzzy control of SISO nonlinear systems with input-output nonlinear relationship," International Journal of Fuzzy Systems, vol. 20, no. 4, pp. 1069-1078, 2018.

[26] S. H. Kim, "Relaxation technique for a T-S fuzzy control design based on a continuous-time fuzzy weighting-dependent Lyapunov function," IEEE Transactions on Fuzzy Systems, vol. 21, no. 4, pp. 761-766, 2013.

[27] Y. L. Hao, ““ Membership-dependent stability conditions for type-1 and interval type-2 T-S fuzzy systems," Fuzzy Sets and Systems, vol. 356, pp. 44-62, 2019.

[28] E. Ramirez, P. Melin, and G. Prado-Arechiga, "Hybrid model based on neural networks, type-1 and type- 2 fuzzy systems for 2-lead cardiac arrhythmia classification," Expert Systems with Applications, vol. 126, pp. 295-307, 2019.

[29] S. Huang, G. Zhao, and M. Chen, "A fast analytical approximation type-reduction method for a class of spiked concave type-2 fuzzy sets," International Journal of Approximate Reasoning, vol. 103, pp. 212-226, 2018.

[30] F. X. Zhang and Y. M. Li, "Indirect adaptive fuzzy control of SISO nonlinear systems with input-output nonlinear relationship," IEEE Transactions on Fuzzy Systems, vol. 99, pp. 1-10, 2018.

[31] A. Baykasoğlu and L. Gölcük, "Development of an interval type-2 fuzzy sets based hierarchical MADM model by combining DEMATEL and TOPSIS," Expert Systems with Applications, vol. 70, no. 15, pp. 37-51, 2017.

[32] A. A. Jafari, S. M. A. Mohammadi, and M. H. Naseriyeh, "Adaptive type-2 fuzzy backstepping control of uncertain fractional-order nonlinear systems with unknown deadzone," Applied Mathematical Modelling, vol. 69, pp. 506-532, 2019.

[33] H. K. Zhang, Y. F. Wang, D. H. Wang, and Y. L. Wang, "Adaptive robust control of oxygen excess ratio for PEMFC system based on type-2 fuzzy logic system," Information Sciences, vol. 511, pp. 1-17, 2020.

[34] S. Xie, Y. Xie, F. Li, Z. Jiang, and W. Gui, "Hybrid fuzzy control for the goethite process in zinc production plant combining type-1 and type-2 fuzzy logics," Neurocomputing, vol. 366, pp. 170-177, 2019.

[35] E. Camci, D. R. Kripalani, L. Ma, E. Kayacan, and M. A. Khanesar, "An aerial robot for rice farm quality inspection with type-2 fuzzy neural networks tuned by particle swarm optimization-sliding mode control hybrid algorithm," Swarm and Evolutionary Computation, vol. 41, pp. 1-8, 2018. 